\title{
BMJ Open Mental health and well-being concerns of fly-in fly-out workers and their partners in Australia: a qualitative study
}

\author{
Benjamin Gardner, ${ }^{1}$ Kristie-Lee Alfrey, ${ }^{2}$ Corneel Vandelanotte, ${ }^{2}$ Amanda L Rebar ${ }^{2}$
}

To cite: Gardner B, Alfrey K-L, Vandelanotte $\mathrm{C}$, et al. Mental health and well-being concerns of fly-in fly-out workers and their partners in Australia: a qualitative study. BMJ Open 2018;8:e019516. doi:10.1136/ bmjopen-2017-019516

- Prepublication history and additional material for this paper are available online. To view these files, please visit the journal online (http://dx.doi org/10.1136/bmjopen-2017019516).

BG and K-LA contributed equally.

Received 7 September 2017 Revised 18 January 2018 Accepted 26 January 2018

Check for updates

${ }^{1}$ Department of Psychology, Institute of Psychiatry,

Psychology and Neuroscience, King's College London, London, UK

${ }^{2}$ School of Health, Medical and Applied Sciences, Central Queensland University, Rockhampton, Queensland, Australia

Correspondence to

Dr Amanda L Rebar;

a.rebar@cqu.edu.au

\section{ABSTRACT}

Objectives Fly-in fly-out (FIF0) work involves commuting long distances to the worksite and living in provided accommodation for 1-4 weeks while on shift. While the potentially detrimental impact of FIFO work on the health and well-being of workers has been documented, little attention has been paid to how workers, or their partners, cope with this impact. This study sought to investigate how workers and their partners negotiate the impact of FIFO on their mental health and well-being.

Design The study design was qualitative. FIF0 workers and partners responded to open-ended questions on concerns about the FIF0 lifestyle and the support they use. Setting Australian FIF0 workers and partners responded to the questions via email.

Participants Participants were 34 FIF0 workers (25 men, $M$ age $=41$ years) and 26 partners of FIF0 workers (26 women, $M$ age $=40$ years).

Results Participant-validated thematic analysis generated three main themes: managing multiple roles, impact on mental health and well-being, and social support needs. Results revealed difficulties in adjusting between the responsibilities of perceptually distinct on-shift and offshift lives, and managing potential psychological distance that develops while workers are on site. Participants emphasised the importance of maintaining quality communication and support from family members. Workers and partners attempted to maintain mental health and well-being by regularly engaging with support networks, although many felt organisational support was tokenistic, stigmatised or lacking.

Conclusions Recommendations for enhancing support provided by FIFO organisations are offered. In particular, organisations should emphasise the importance of good mental health and well-being, maintain transparency regarding potential challenges of FIFO lifestyles, and offer professional support for managing multiple social roles and effective communication.

With unique work shifts come unique lifestyle situations. Fly-in fly-out (FIFO; also known as drive-in drive-out) work involves employees travelling long distances to the worksite, living in provided accommodation during their on-shift roster and travelling home between shifts. ${ }^{1}$ FIFO workers commonly have schedules of 12-hour shifts for 1-4 consecutive weeks. $^{2}$ Also termed long-distance

\section{Strengths and limitations of this study}

This is the first study to our knowledge to have explored the impact of long-distance commuting on both fly-in fly-out (FIF0) workers and partners.

- Study participants were situated across Australia, including those at FIFO sites.

- We collected data via questions probing domains that we deemed to be important to FIF0 workers and their partners. These questions may have neglected other relevant areas of the FIFO experience relevant to health and well-being.

- While our participants cited various adverse impacts of FIFO work on their health and well-being, we cannot identify the mechanisms underlying such impact.

commuting, FIFO work is becoming increasingly prevalent in Australia, mostly as a result of the mining industry boom of the last 15 years, although it is also common in the construction and resource sectors. ${ }^{3}$ Although FIFO work is still relatively rare, in some Western Australian and Queensland communities, as many as one-in-six people are employed in FIFO positions. ${ }^{14}$ Concerns have been raised around the health and well-being impact of FIFO work, ${ }^{56}$ but evidence around the impact on mental health and well-being of workers and their partners is in its infancy.

Most FIFO workers are young or middleaged men, a demographic already particularly prone to mental health problems and at increased risk for suicide. ${ }^{4}$ Industry reports have suggested that there are few disadvantages to FIFO life other than potential inconvenience of prolonged work shifts, and that there are many mental health benefits, including being part of a challenging work environment, and unique opportunities to meet new people, see new places and earn a high income. ${ }^{58}$ However, research suggests that FIFO work has both costs and benefits for mental health and well-being. ${ }^{9} 10$ For example, Torkington et $a l^{11}$ interviewed 11 FIFO workers about their psychosocial 
well-being and perceived support. Some found their job rewarding and enjoyed interactions with colleagues, but others experienced loneliness, fatigue and problems in balancing time away for work with social and family time. Other research has suggested that, among workers with long shifts and low autonomy over their shift schedules, FIFO work can have negative repercussions for both work and home life. ${ }^{12}$ Such problems may be compounded by a failure to access support; relative to non-FIFO workers, FIFO workers have also been found to be less likely to report or seek help for mental health concerns. ${ }^{13}$

FIFO work may also impact on the well-being of workers' significant others. Most research on the impact of FIFO work on mental health or well-being has centred on workers' children. While one study ${ }^{14}$ found no differences between FIFO families and non-FIFO families in relationship quality, parenting competence, or child emotional and behavioural difficulties, another ${ }^{15}$ showed that adolescent children's depressive symptoms and emotional and behavioural difficulties could be partially attributed to the intermittent parental absence that characterises FIFO employment. While these findings suggest that the extent of impact of FIFO work on mental health may vary depending on the people involved and the home and work contexts, they nonetheless point to the potential for FIFO work to impact on family members.

Kaczmarek and Sibbel ${ }^{16}$ found that the well-being of FIFO workers' primary school-aged children did not significantly differ from that of similar families with a parent in the military or from the general community. Yet partners of the FIFO workers in this study reported more problems with communication, support and behaviour control within the family than did families from the military or the general community. Quantitative survey findings from Israel and the USA suggest that FIFO work can have a modest negative impact on couples' relationship satisfaction. ${ }^{17}$ A case study in Canada found that FIFO couples can face numerous challenges, including transitioning between on-shift and off-shift roles and parenting. ${ }^{18}$

A study of people who had committed suicide compared Australian miners (of whom many were FIFO workers) with non-miners, and found that the miners were significantly more likely to have experienced relationship problems. ${ }^{19}$ This demonstrates the potential interdependence of the mental health and well-being of FIFO workers and their partners, and the complex and dynamic impact of FIFO work on workers and others.

The unique lifestyle circumstances imposed by FIFO work have been associated with potential mental health risks. Yet little evidence exists regarding how best to support FIFO workers and partners to navigate the complexities of FIFO life. Some evidence suggests that family cohesion, connectedness, flexibility and meaningful communication are important factors for buffering from potential negative effects of FIFO life on well-being. ${ }^{20}{ }^{21}$ Quality family time, routines, social support networks and clear set boundaries also aid in the adjustment and management of the FIFO lifestyle. ${ }^{20}$ However, beyond this general evidence about how families might cope with FIFO life, little has been documented regarding how workers and partners can manage FIFO work to maintain positive mental health and well-being.

\section{THE PRESENT STUDY}

The aim of this study was to develop understanding of how FIFO workers and their partners experience and negotiate the impact of FIFO work on their mental health and well-being. Understanding how workers and partners manage any negative consequences of the FIFO lifestyle may be informative for intervention purposes, because it may reveal useful coping strategies, while identifying areas in which support may be especially required. Qualitative research methods were used to obtain a rich and indepth insight into participants' experiences.

\section{METHODS}

\section{Participants}

Study recruitment was conducted with convenience sampling through FIFO-relevant online social media group pages and media outlets of regional Australian audiences (eg, radio, television, newspapers, websites). Eligibility was not contingent on both partners of a couple being involved in the study, making it possible that the partner of a FIFO worker may have participated despite the worker himself or herself not doing so, and vice versa. In return for their involvement, participants were entered into a random draw for $\$ A 30$ (US\$24) gift vouchers, a value which we deemed to be motivating, but not coercive, for potential participants. No a priori sample size requirements were set.

\section{Procedures}

Participants self-reported their age, sex and their (or their partner's) FIFO working patterns (eg, roster length) through an online survey. They were also asked whether overall, they liked (their partner) being a FIFO worker or not (yes/no). Participants were then asked to respond to a set of questions about the FIFO lifestyle via email. We chose to collect data via email to gain access to FIFO workers and partners situated across Australia, including those at FIFO sites, with minimal inconvenience to participants. Additionally, the email-based survey may have allowed participants to feel less identifiable when responding about potentially stigmatising mental health issues than is possible with face-to-face interviews.

The questions were developed for the purposes of this study and the full list of questions is available as online supplementary file 1. Example questions include 'Are you concerned about how the FIFO lifestyle affects you?' and 'Do you have suggestions on how support for FIFO workers and FIFO partners could be made better?' Although these were not open questions, participants were invited to provide free-text (rather than yes/no) responses, and 
all participants did so. The terms 'mental health' and 'wellbeing' did not feature in the questions to minimise potential self-presentational concerns inhibiting disclosure of relevant issues. All participants provided informed consent prior to participating in the study.

\section{Analyses}

Responses were analysed by one researcher using thematic analysis procedures, ${ }^{22}$ based on realist epistemological assumptions. The analyst was a UK-based social and health psychologist with expertise in qualitative analysis (BG), who has no personal links to FIFO, no history of research in this domain and was unfamiliar with the FIFO research literature prior to and during the analysis. The analyst was recruited to the research team after data had been collected to minimise the possibility that the analysis would be influenced by preconceptions of FIFO or experiences of data collection. Responses were read and reread, for familiarisation purposes. Line-by-line coding was undertaken to assign conceptual labels to pertinent excerpts. As coding progressed, an inductively derived thematic framework was developed and iteratively refined to best reflect emergent insights. Themes were labelled in part using representative phrases (ie, 'in vivo' codes) taken verbatim from the data, to demonstrate the veracity of the theme. ${ }^{23}$ A second researcher (ALR) inspected the final coding framework and analysis, and verified that the themes were coherent representations of the data. The final narrative was also verified, by two FIFO workers and three partners who participated in the study, as a valid conceptual analysis of the FIFO experience.

Data excerpts are quoted below as evidence of the validity of the analysis. ${ }^{24}$ To aid clarity where necessary, punctuation was added, spelling mistakes corrected and words added in brackets to clarify intended meaning.

\section{RESULTS}

The final data set comprised 34 FIFO workers (25 men $(79 \%), 9$ women $(21 \%), M$ age $=41$ years, $\mathrm{SD}=11$, age range $=25-65$ years) and 26 partners of FIFO workers (all women, $M$ age $=40$ years, $\mathrm{SD}=9$, age range $=27-58$ years ). The sample included six couples (ie, six workers, six partners). The remaining 48 participants (28 FIFO workers, 20 partners) took part in the study without the involvement of their partners. No participant withdrew from the study.

\section{Sample description}

FIFO workers most commonly worked either day shifts $(48 \%)$ or a mixture of day and night shifts $(48 \%)$. Only one worker exclusively worked night shifts. Workers' rosters were between 4 and 29 workdays on shift $(M=15$, $\mathrm{SD}=8)$, with between 2 and 21 days off shift $(M=8, \mathrm{SD}=4)$. More than half $(62 \%)$ of workers reported that overall they liked being a FIFO worker. Partners reported that their partners mostly worked day shifts (62\%), with some working a mixture of day and night shifts (35\%), and one person working night shifts. Partners reported their FIFO worker partners to work between 6 and 60 workdays on shift $(M=20, \mathrm{SD}=12)$, with between 5 and 21 days off shift $(M=8, \mathrm{SD}=4)$. More than half $(64 \%)$ of FIFO partners reported that overall they did not like that their partner was a FIFO worker. On average, participants' household income was \$A182481 ( US\$143 000; $\mathrm{SD}=\$ \mathrm{~A} 56905$ (US\$44700)), with the range between $\$ A 52000$ and $\$ A 320000$ (US\$41 000-US\$250000). Workers' occupations included plant operators, managers, train drivers, heavy machinery operators and specialists.

\section{Thematic analysis}

Three themes were extracted, relating to experiences of negotiating multiple social roles (theme 1), health and well-being issues surrounding FIFO employment (theme 2), and social support needs (theme 3 ).

\section{Theme 1: "I'm leading two lives": managing multiple roles}

FIFO workers typically conceived of their work and home lives as two discrete 'worlds', characterised by different lifestyles, roles and responsibilities ("I'm leading two personalities and two lives", participant 13 (P13), worker, male (M), 38 years old). Commonly described through a contrast with the domestic 'world', the FIFO 'world' was seen to be more rigidly structured, but allowing greater personal freedom, due to provision of assistance for everyday domestic activities and the absence of immediate family commitments ("I don't have to worry about cooking, cleaning etc., " P11, worker, M, 34 years old). The demands of these two 'worlds' necessitated the adoption of different social roles and patterns of behaviour:

At work I have enormous pressure to deal with so [I am] more aggressive and business-oriented. I need to maintain a bravado in a male-dominated industry. At home I have to be happy, supportive, caring, friendly and show empathy. (P13, worker, M, 38 years old)

While away I can just be a bloke. [When I'm at] home I'm a family man. (P22, worker, M, 47 years old)

Workers' partners also described two 'worlds', with the enhanced burden of domestic duties and responsibilities imposed when workers are away requiring greater self-sufficiency ("[she's] almost [a] pseudo single-parent, in certain circumstances," P26, worker, M, 52 years old).

My wife [says] "when he is away, I have to be strong and independent, service the car, change the light bulbs, but when he comes home I am weak, defenceless and dependent". (P32, worker, M, did not report age)

Several workers experienced difficulties in negotiating the transition between their two 'worlds' when returning home from a shift, struggling to adjust to differences in the pace and requirements of domestic life: 
The first few days [back home involve] trying to get up to speed with day to day life, and a different routine. (P19, worker, M, 42 years old)

It is sometimes difficult to readjust and function as an adult at home. By the time you have adjusted, it is time to fly out again. (P5, worker, M, 28 years old)

Similarly, some partners struggled to adjust their settled domestic routines to incorporate workers' return home, which was a potential source of tension:

When my partner comes home he feels like an outsider, as the kids and myself are in a routine that differs from him. He tries to change things into his way of doing, which creates havoc in the household. (P50, partner, female $(\mathrm{F}), 43$ years old)

Participants described a process of renegotiating domestic roles and responsibilities on workers' return, with some partners expressing frustration at FIFO workers for not assuming greater domestic responsibility:

I work 3-5 days a week [and] I get mad at [him] sometimes as he is home and not helping with household duties. (P43, partner, F, 36 years old)

\section{Theme 2: "The FIFO roster was the breaking point": impact on} mental health and well-being

For most workers and partners, financial gain was the primary benefit of FIFO employment ("I am only working this lifestyle to get ahead financially", P1, worker, M, 23 years old). Income was a source of stress for some however, as they felt they had limited autonomy over their employment and career, having become "trapped" into undesirable working patterns by becoming accustomed to high income ("I am locked into this lifestyle now", P57, partner, $\mathrm{F}, 57$ years old):

The golden handcuffs go on. As people earn more, they spend more, and take on larger debt burdens, causing them to be trapped in the mining FIFO work lifestyle. (P7, worker, M, 32 years old)

Indeed, many participants described adverse mental health and well-being effects of FIFO employment. For many, absence from family was particularly detrimental. Workers, particularly those with children, often felt that they were missing out on potentially significant family events ("I have missed out on a lot of living and memories with family", P57, worker, M, 57 years old). Both workers and partners worried about the impact of the prolonged absence of one parent on children's well-being and development ("Will we have regrets later? Are they missing out on more than we realise, having their Dad work away?" $\mathrm{P} 42$, partner, F, 34 years old). Workers also voiced concerns about being unable to respond to domestic emergencies while on shift:

We only have two flights here every week, Monday and Thursday. Once that window to escape closes, you are trapped, and constantly hoping that nothing happens back home. (P18, worker, M, 42 years old)

Many participants felt that FIFO work put considerable strain on relationships with partners. Many spoke of physical separation leading to a sense of psychological distance, such that they felt "disconnected" (P51, partner, F, 44 years old) or were "leading separate lives" (P28, worker, M, 58 years old). Communication between workers and their partners was valued as a means of maintaining relationships, but distance was often felt to reduce the quality of such communication:

We talk every day, although I sometimes struggle to remain interested sometimes as she is not in front of me, merely a voice on the phone. It can be a struggle to bring up subjects of conversation, as my day can be quite mundane yet her job as a teacher can have so many events happen that she wants to tell me about. ( $\mathrm{P} 6$, worker, $\mathrm{M}, 29$ years old)

[The FIFO lifestyle] adds strain when we are both tired. If we were home we would say nothing and hug but that's not possible through the telephone so it makes for awkward phone calls. (P37, partner, F, 27 years old)

Physical and psychological distance was reportedly a source of tension for many. Some participants reported growing suspicious of their partner's fidelity ("II worry that] he'll get bored and cheat on me", P41, partner, F, 33 years old), and others felt resentful towards their partners, for failing to fully acknowledge the perceived sacrifices each makes for the family unit:

Absence doesn't make the heart grow fonder. When things get tough at home, the resentment can sometimes creep in. ( $\mathrm{P} 46$, partner, $\mathrm{F}, 38$ years old)

I do resent the fact that he has a week off where he gets to do nothing. I am working full time and raising our small child, which means I have been unable to attend a number of training and workshop sessions for my career advancement due to childcare issues when he is at work. (P47, partner, F, 38 years old)

Perhaps consequently, many participants described deterioration or dissolution of relationships:

The FIFO roster was eventually the breaking point of our relationship. It's hard to expect a partner to be okay with a half time person in a relationship. (P10, worker, F, 34 years old)

Many workers described feelings of isolation and loneliness due to prolonged absence from their families, which for some reportedly manifested in anxiety or depression:

My family feels safe when I'm home, I'm not lonely. I don't [have] anxiety when I'm home. (P24, worker, M, 55 years old)

Partners also described feelings of emotional strain: 
My concerns would be the impact it's had on my mental state of mind at times. Raising three children on my own hasn't been easy. At times, you feel like you can't go on. (P40, partner, F, 33 years old)

The impact of FIFO on partners was an additional concern for many. Several workers reported feeling guilty for delegating everyday domestic duties and responsibilities to their partners ("IIt] puts stress on my wife. She's effectively a single mum for 2 weeks out of every three", P51, worker, M, 44 years old), while partners worried about workers' physical and mental health ("Is he getting enough sleep, eating correctly, not drinking too much?", P43, partner, F, 36 years old).

\section{Theme 3: "Others don't understand how hard it is": social support} needs

Workers and partners generally felt unsupported in negotiating health and well-being problems associated with FIFO employment. Many participants felt that people not involved in FIFO work lack sympathy and believe that the high income disqualifies any detrimental impacts:

It's difficult to help others understand how hard it is. [...] There's a perception that it's the perfect lifestyle so why should FIFO workers complain. (P13, worker, M, 38 years old)

Some participants were also unsympathetic to fellow FIFO workers, attributing causality for health and wellbeing problems to bad decision-making by workers:

[FIFO workers] need to think about what the job involves and stop blaming everyone else when things get tough. They weren't made to take the job. [They] need to [stand] back and look at themselves and reassess their situation. (P8, worker, M, 33 years old)

Most participants reported receiving most support from their partners ("we communicate very well, we always support and encourage one another when times are tough, and know when to give that support", P40, partner, F, 33 years old). However, a mutual lack of shared experience meant that many workers felt that partners did not fully appreciate the impact of FIFO working, and conversely many partners felt that workers did not fully appreciate the impact of an increased domestic burden:

Partners need to understand the stress workers are faced with, being away and then being home. (P27, worker, $\mathrm{F}, 55$ years old)

I have tried talking to my partner about how I feel and he cannot see my problem. (P56, partner, F, 56 years old)

Many workers and partners felt that FIFO employers were unsupportive, prioritising productivity over workers' health and well-being, and offering only tokenistic support:

They don't always want to accept the responsibility. They preach all the stuff at inductions [but] when it comes to applying it they turn a blind eye. (P3, worker, M, 26 years old)

Employers spout about mental health, but are not lenient when concessions need to be made for people with mental health issues. (P43, partner, F, 36 years old)

Many workers were reluctant to seek help for health or well-being issues. Some reported not always being able to recognise when they required help ("I had a stage where I was down and I didn't even know it", P4, worker, M, 27 years old), as mental health issues were common among workers ("the struggles they face are what everyone else is feeling too", P6, worker, M, 29 years old). Some did not prioritise help-seeking, instead preferring to "try to tough things out" (P30, worker, M, 61 years old). Others reported a 'macho' culture in which help-seeking was viewed as a display of weakness, and felt that seeking help could cost them their job:

There is still some stigma attached to getting help due to the 'manly' side of sucking it up and getting on with the job. Those that have issues either keep it to themselves or are labelled as not being able to cut it. (P26, worker, M, 52 years old)

There is a bit of concern among workers that this [support] service is tracked by the employer who uses it, and this may be a black mark against the person using the service. The fear of losing your job because of mental health concerns is still very relevant in mining. (P7, worker, $\mathrm{M}, 32$ years old)

Workers felt that greater acknowledgement and empathy from management would encourage more help-seeking:

The stigmas still surrounding mental health issues in mining prevent people accessing services on site. If this culture was to improve and promote mental health as a major health and safety topic in the workplace where people are comfortable talking about it openly, this would be the main way to improve support for workers. (P7, worker, M, 32 years old)

Other suggestions offered by workers for improved support from employers included providing dedicated support workers or a 'buddy system' for discussing health, greater choice of shift patterns and facilitating close communication with family:

Adequate communication infrastructure should be available to the people on site so that partners can contact them at any time and vice versa. (P26, worker, M, 52 years old)

Site visits [for family members] need to be more readily available. It would help the families at home to see what their loved one goes away to. (P12, worker, F, 37 years old)

Several partners reported gaining social support through membership of specialist online social networks 
( "Facebook has FIFO-wife pages, which offer great support and advice", P50, partner, F, 43 years old). Connecting with others with similar experiences was felt to validate partners' concerns ( 'it just gives you relief, knowing you're not the only one having a crappy night or day", P41, partner, F, 33 years old). Two partners, however, felt that online support networks should be administered and moderated by employers, having faced hostility from others in an informal FIFO social network ("I asked for some support ... [and] I was brutally attacked by other members. I quickly deleted myself from the group", P59, partner, F, 58 years old).

\section{DISCUSSION}

Enhancing positive mental health and well-being in the workplace is recognised by the WHO as a global research priority. ${ }^{25}$ This study explored reflections among FIFO workers and their partners on the mental health and well-being impact of FIFO work and strategies for mitigating these concerns. FIFO work is characterised by prolonged periods of working long daily hours away from home. ${ }^{2}$ Workers commonly reported difficulty in adjusting between their on-shift and off-shift roles and responsibilities. Both workers and partners spoke of the development of psychological distance between workers and their partners and the strain this placed on relationships. Feelings of isolation and loneliness were prevalent, along with concerns of how FIFO work impacted communication between workers and their partners. Workers and partners alike typically felt unsupported. There was scepticism of, and reluctance to access, support provided by FIFO organisations, as well as a general feeling that the general public is unsympathetic towards FIFO workers and families. Strategies deemed useful for mitigating problems associated with FIFO work patterns included maintaining effective communication with partners, and receiving emotional and practical support from family members, neighbours and other FIFO families. These findings provide a unique insight into the methods used by workers and partners to navigate the adverse impacts of FIFO and point to areas in which additional support may be needed.

Several aspects of the FIFO lifestyle were seen by our participants as potential threats to mental health or wellbeing. Some workers reported feeling "trapped", such that they were unhappy in FIFO work but felt unable to take lower paying alternative employment, having grown accustomed to the high-income levels provided by FIFO. The implicit trade-off between financial constraints and job satisfaction is likely to compromise worker wellbeing. These findings are in line with previous FIFO work research findings that job satisfaction and perceived autonomy over career decisions are important determinants of workplace well-being. ${ }^{26}{ }^{27}$ Participants did not report the methods that they use to seek to overcome feelings of psychological entrapment. Nonetheless, theory proposes that people are likely to feel more intrinsically motivated in work that provides feelings of autonomy, competence and relatedness. Thus, we recommend that FIFO organisations seek to reduce these feelings of externally regulated impositions through simple changes in the work climate to enhance feelings of self-determination, competence and a meaningful social connection to others arising from FIFO work..$^{28}$

Workers also reported difficulty in balancing the demands of FIFO working patterns with domestic commitments. Many reported being unable to achieve a work-life balance. This is often seen by workers to be the main disadvantage of FIFO work. ${ }^{11} 1226$ Our participants commonly conceived of 'work' and 'life' as 'separate worlds', characterised by different social roles, expectations and patterns of behaviour. While the perceptual 'work'-'life' distinction is not specific to FIFO (eg, ref 29), work-life transitional issues may perhaps be more pronounced, or have greater impact, among those working long hours or for prolonged periods away from home. ${ }^{30}$ Partners also described challenges in adjusting their domestic routines according to the presence or absence of FIFO workers. Both workers and partners spoke of periods of disruption as they struggled to adjust to changes in established routines. Participants did not report how they overcame these challenges. Training in maintaining a comfortable work-life balance, and negotiating work-life transitions, may perhaps be useful for FIFO workers and their partners. ${ }^{31}$

For many participants, geographical distance, and the regular and prolonged absence of FIFO workers led to psychological detachment of workers from their families. Similar experiences have been documented among other long-distance commuters, such as long-haul truckers and commercial fishermen. ${ }^{18}{ }^{32}$ This is likely to have multiple adverse effects on well-being. Many participants felt isolated and lonely, a common experience among FIFO workers. ${ }^{33}$ Workers may also miss out on shared social experiences and feelings of companionship, which have been shown to buffer against the adverse impact of everyday life stressors. ${ }^{34}$ Modern advances in communication (eg, video calls, social media) may help reduce, but not fully alleviate, some of the concerns of geographical distance for FIFO workers and their partners. ${ }^{18}$ FIFO organisations might alleviate these feelings of loneliness and enhance social support for mental health by providing workers access to structured opportunities for social contact (eg, community-based recreation groups).

Psychological distance also reportedly adversely affected the quality of relationships between workers and their partners, potentially leading to tension and distrust. Some participants were able to mitigate these impacts by maintaining close communication with partners. Indeed, effective communication is a characteristic of cohesive and well-functioning families. ${ }^{21}$ Yet some workers felt unable to effectively communicate with their partners, citing either a lack of shared experiences to discuss or a lack of adequate on-site communication infrastructure. FIFO organisations should acknowledge the importance of regular communication for maintaining relationships 
by prioritising the provision of access to timely and private contact between on-shift workers and their families. Alternatively, as some participants suggested, organisations might allow families to visit FIFO sites. While costly to administer, research suggests that on-site 'family days' provide partners with insight into workers' roles and responsibilities, such that they are better able to understand and empathise with workers' experiences and concerns. ${ }^{35}$ Workers, too, report that such initiatives make them feel valued and supported by employers. ${ }^{35}$

Many participants felt that they lacked social support, which made it difficult to negotiate the challenges posed by FIFO work. Previous research attests to the importance of social support for maintaining mental health, especially in situations of high stress. ${ }^{3637}$ Our participants perceived the public to be unsympathetic to FIFO workers and their families, and indeed previous research has highlighted negative media portrayals of FIFO workers as greedy and undeserving. ${ }^{38}$ While participants acknowledged that support was available, many were reluctant to access it, citing stigma around seeking support for mental health. While stigma surrounding mental health and help-seeking is well-documented, ${ }^{39}$ this may be especially pronounced in the typically male-oriented FIFO domain, ${ }^{4}$ as norms of masculinity may further inhibit help-seeking for mental health. ${ }^{40}$ Some partners reported having obtained support using online social networks. Previous research has shown the benefits of online support: while online forums should not be seen as a substitute for professional mental health services, $75 \%$ of users of one Norwegian forum found it easier to obtain support from an online forum than to discuss mental health problems in person. ${ }^{41}$ User anonymity afforded by online forums can disinhibit help-seeking. ${ }^{42}$ Conversely, however, anonymity can also facilitate antisocial behaviour; two partners in our sample reported receiving abuse from members of an online forum. FIFO organisations should consider funding professionally moderated online support networks for FIFO workers and their families to minimise such problems. Professional involvement can also minimise the possibility that users become dependent on the support of other forum members and withdraw from inperson contact. ${ }^{42}$ Furthermore, the administration of social support networks by FIFO organisations, or professionals allied to FIFO organisations, would allow for the integration of structured activities conducive to mental health given the issues raised by FIFO workers. This is important, because participation in shared activities fosters a sense of control, belonging, self-esteem and social support. These in turn can buffer against mental health problems, just as strongly as can the sharing of thoughts and feelings with others. ${ }^{37}$

Many workers did not feel supported by employers and were sceptical of the motives of FIFO organisations, viewing them as ultimately unsympathetic to the mental health and well-being needs of workers. Available support was viewed as tokenistic, and most workers felt that their jobs would be under threat if they attempted to access support. This is problematic, as organisational support (both actual and perceived) is central for achieving health and well-being in the workplace $\left(\mathrm{eg}, \mathrm{ref}{ }^{43}\right)$. Cynicism towards workplace health policy can arise from perceptions of senior management as lacking integrity, competence or trustworthiness. Cynicism may be overcome by adopting a more participatory approach to the development and implementation of mental health support. ${ }^{44}$ Employee involvement in workplace health policy development would allow for integration of workers' experience and knowledge, and may build trust between management and employees, so achieving greater acceptance among the FIFO workforce.

\section{Limitations and future directions}

Limitations of our study must be acknowledged. We collected data via questions probing domains that we deemed to be important to FIFO workers and their partners. These questions may have neglected other areas of the FIFO experience relevant to health and well-being. Additionally, our survey design, whereby qualitative data were collected via online free-text responses, did not allow us to probe further into participants' responses. While our participants cited various adverse impacts of FIFO work on their health and well-being, we cannot identify the mechanisms underlying such impact. It is plausible, for example, that FIFO poses risks to mental health and well-being only among workers with low job satisfaction or perceived autonomy. ${ }^{22}$ Additionally, while we sought to document participants' coping strategies, in some instances-for example, where describing feelings of psychological entrapment—no such strategies were cited. Semistructured interviews, in which participants can be asked to expand on their responses, may have produced a deeper insight into the issues we documented, and future such research will be important for elaborating on these study findings. Furthermore, our sample size was relatively small, and the generalisability of findings is unclear. There are likely unique mental health and well-being concerns for FIFO workers in different regions, roster lengths and occupations. ${ }^{17}$ Our data may have been influenced by selection bias, such that those who were most motivated to respond to our survey were those with the most negative experiences. While the veracity of our analysis was confirmed by a subsample of our participants, it is unclear whether the views documented among our sample are representative of FIFO workers, or their partners more broadly. However, our aim was not to generate generalisable findings, but rather to document health and well-being experiences pertinent to FIFO workers and their partners. Indeed, ours is the first study to our knowledge to have explored the impact of FIFO on partners. Our findings highlight the need for the provision of support to both workers and their partners.

This study highlighted the mental health and well-being concerns raised by FIFO workers and their partners, and the strategies they used to address these concerns, while pointing to areas in which further support may be 
needed. FIFO organisations may need to acknowledge, in a manner more visible and transparent to employees, the importance of worker health and well-being, and offer unconditional support to address their concerns. While many felt unsupported, some workers and their partners were able to mitigate the potential adverse impact of FIFO by maintaining close communication with partners, and securing emotional and practical support from others. FIFO organisations, and their employees, may benefit from implementing workplace health and well-being programmes codesigned by management and employees to address these concerns.

Contributors K-LA, CV and ALR helped conceive of the idea of the study design, collected the data and provided intellectual content for the manuscript. BG conducted the data analysis, assisted in interpreting the findings and provided intellectual content for the manuscript. All authors were involved in drafting the manuscript and revising it critically for important intellectual content, and gave approval of the final version to be published.

Funding Participant incentives were donated by the not-for-profit organisation, LIVIN (Australia). They had no other involvement in the study procedures, analyses or dissemination.

Competing interests None declared.

Patient consent Not required.

Ethics approval All study procedures were approved a priori by the Central Queensland University's Human Research Ethics Committee.

Provenance and peer review Not commissioned; externally peer reviewed.

Data sharing statement Extra data are available by emailing the corresponding author (ALR).

Open Access This is an Open Access article distributed in accordance with the Creative Commons Attribution Non Commercial (CC BY-NC 4.0) license, which permits others to distribute, remix, adapt, build upon this work non-commercially, and license their derivative works on different terms, provided the original work is properly cited and the use is non-commercial. See: http://creativecommons.org/ licenses/by-nc/4.0/

(C) Article author(s) (or their employer(s) unless otherwise stated in the text of the article) 2018. All rights reserved. No commercial use is permitted unless otherwise expressly granted.

\section{REFERENCES}

1. Australian Bureau of Statistics. Fly-in Fly-out (FIFO) workers. Canberra, Australia: Australian Institute of Health and Welfare, 2014.

2. De Silva H, Johnson L, Wade K. Long distance commuters in Australia: a socio-economic and demographic profile. Australia: The Department of Infrastructure, Regional Development and Cities, 2011.

3. Australian Bureau of Statistics. Towns of the mining boom. Canberra, Australia: Australian Institute of Health and Welfare, 2013.

4. Education and Health Standing Committee. The impact of FIFO work practices on mental health. Perth, WA, Australia: Legislative Assembly, Parliament of Western Australia, 2015.

5. House of Representatives Standing Committee on Regional Australia. Cancer of the bush or salvation for our cities?: Fly-in, flyout and drive-in, drive-out workforce practices in regional Australia. Canberra, Australia: Common wealth of Australia, 2013.

6. Solomon F, Katz E, Lovel R. Social dimensions of mining: Research, policy and practice challenges for the minerals industry in Australia. Resources Policy 2008;33:142-9.

7. Misan GM, Rudnik E. The pros and cons of long distance commuting: Comments from South Australian mining and resource workers. J Econ Soc Policy 2015;17:6-37.

8. The Chamber of Minerals and Energy: Western Australia. Fly In / Fly out: a sustainability perspective: a discussion of thetriple bottom line impact of fly-in / fly-out operations in Western Australia. Perth, WA, Australia: The Chamber of Minerals and Energy, 2005.

9. Lewis J, Shrimpton M, Storey K. Family members' experience of offshore oil work in New foundland. Women, work and family in the
British, Canadian and Norwegianoffshore oilfields. London: Palgrave Macmillan, 1988:163-89.

10. Ryser L, Schwamborn J, Halseth G, et al. Working 'away': Community and family impacts of long distance labour commuting in Mackenzie, BC: Final report. Prince George, BC: Community Development Institute University of Northern British Columbia, 2011.

11. Torkington AM, Larkins S, Gupta TS. The psychosocial impacts of fly-in fly-out and drive-in drive-out mining on mining employees: a qualitative study. Aust J Rural Health 2011;19:135-41.

12. Knox A, Warhurst $C$, Pocock $B$, et al. 'You get really old, really quick': involuntary long hours in the mining industry. $J$ Ind Relat 2011;53:13-29.

13. Joyce SJ, Tomlin SM, Somerford PJ, et al. Health behaviours and outcomes associated with fly-in fly-out and shift workers in Western Australia. Intern Med J 2013;43:440-4.

14. Dittman CK, Henriquez A, Roxburgh N. When a Non-resident worker is a non-resident parent: investigating the family impact of fly-in, flyout work practices in Australia. J Child Fam Stud 2016;25:2778-96.

15. Lester L, Watson J, Waters S, et al. The association of fly-in flyout employment, family connectedness, parental presence and adolescent wellbeing. J Child Fam Stud 2016;25:3619-26.

16. Kaczmarek EA, Sibbel AM. The psychosocial well-being of children from Australian military and fly-in/fly-out (FIFO) mining families. Community Work Fam 2008;11:297-312.

17. Landesman J, Seward RR. Long distance commuting and couple satisfaction in Israel and United States: An exploratory study. $J$ Comp Fam Stud 2013;44:765-81.

18. Whalen $\mathrm{H}$, Schmidt $\mathrm{G}$. The women who remain behind: challenges in the LDC lifestyle. Rural Society 2016;25:1-14.

19. McPhedran S, De Leo D. Suicide among miners in Queensland, Australia: a comparative analysis of demographics, psychiatric history, and stressful life events. SAGE Open 2013;3:2158244013511262

20. Lester L, Waters S, Spears B, et al. Parenting adolescents: developing strategies for FIFO parents. J Child Fam Stud 2015;24:3757-66.

21. Taylor J, Simmonds J. Family stress and coping in the fly-in fly-out workforce. Aust Community Psychol 2009;21:23-36.

22. Braun V, Clarke V. Using thematic analysis in psychology. Qual Res Psychol 2006;3:77-101.

23. Glaser B, Strauss A. The discovery of grounded theory: strategies for qualitative research. Chicago: Aldin Pub. Co, 1967.

24. Mays N, Pope C. Rigour and qualitative research. BMJ 1995;311:109-12.

25. World Health Organization. The World health report 2002: reducing risks, promoting healthy life. Geneva, Switzerland: World Health Organization, 2002.

26. Barclay MA, Harris J, Everingham J-A, et al. Geologists, FIFO work practices and job satisfaction. Applied Earth Science 2016;125:221-30.

27. Ryan RM, Deci EL. Self-determination theory and the facilitation of intrinsic motivation, social development, and well-being. Am Psychol 2000;55:68-78.

28. Stone DN, Deci EL, Ryan RM. Beyond talk: creating autonomous motivation through self-determination theory. Journal of General Management 2009;34:75-91.

29. Frone MR, Russell M, Cooper ML. Antecedents and outcomes of work-family conflict: testing a model of the work-family interface. $J$ Appl Psychol 1992;77:65-78.

30. Major VS, Klein KJ, Ehrhart MG. Work time, work interference with family, and psychological distress. J Appl Psychol 2002;87:427-36.

31. Gee Wilson M, Polzer-Debruyne A, Chen S, et al. Shift work interventions for reduced work-family conflict. Employee Relations 2007;29:162-77.

32. Zvonkovic AM, Solomon CR, Humble AM, et al. Family work and relationships: lessons from families of men whose jobs require travel. Fam Relat 2005;54:411-22.

33. Carter T, Kaczmarek E. An exploration of generation Y's experiences of offshore fly-in/fly-out (FIFO) employment. Aust Community Psychol 2009;21:52-66.

34. Kleiber DA, Hutchinson SL, Williams R. Leisure as a Resource in Transcending Negative Life Events: Self-Protection, Self-Restoration, and Personal Transformation. Leis Sci 2002;24:219-35.

35. Goater S, Goater R, Goater I, et al. This life of mine: personal reflections on the well-being of the contracted fly-in, fly-out workforce. 2012 http://www.academia.edu/download/33505950/ Goater_2012_This_life_of_mine_AUSIMM.pdf

36. Kessler RC, McLeod JD. Social support and mental health in community samples. In: Cohen S, Syme SL, eds. Social support and health. San Diego, CA: Academic Press, 1985:219-40. 
37. Thoits PA. Mechanisms linking social ties and support to physical and mental health. J Health Soc Behav 2011;52:145-61.

38. Pini B, McDonald P, Mayes R. Class contestations and Australia's resource boom: the emergence of the 'cashed-up bogan'. Sociology 2012;46:142-58.

39. Clement $\mathrm{S}$, Schauman $\mathrm{O}$, Graham T, et al. What is the impact of mental health-related stigma on help-seeking? A systematic review of quantitative and qualitative studies. Psychol Med 2015;45:11-27.

40. Vogel DL, Heimerdinger-Edwards SR, Hammer JH, et al. "Boys don't cry": examination of the links between endorsement of masculine norms, self-stigma, and help-seeking attitudes for men from diverse backgrounds. J Couns Psychol 2011;58:368-82.
41. Kummervold PE, Gammon D, Bergvik S, et al. Social support in a wired world: use of online mental health forums in Norway. Nord $J$ Psychiatry 2002;56:59-65.

42. Barak A, Boniel-Nissim M, Suler J. Fostering empowerment in online support groups. Comput Human Behav 2008;24:1867-83.

43. Hadgraft NT, Brakenridge CL, LaMontagne AD, et al. Feasibility and acceptability of reducing workplace sitting time: a qualitative study with Australian office workers. BMC Public Health 2016;16:933.

44. Grawitch MJ, Gottschalk M, Munz DC. The path to a healthy workplace: a critical review linking healthy workplace practices, employee well-being, and organizational improvements. Consulting Psychology Journal: Practice and Research 2006;58:129-47. 